\title{
VECTOR VARIATIONAL PROBLEMS AND APPLICATIONS TO OPTIMAL DESIGN
}

\author{
PABlo PEDREGal ${ }^{1}$
}

\begin{abstract}
We examine how the use of typical techniques from non-convex vector variational problems can help in understanding optimal design problems in conductivity. After describing the main ideas of the underlying analysis and providing some standard material in an attempt to make the exposition self-contained, we show how those ideas apply to a typical optimal desing problem with two different conducting materials. Then we examine the equivalent relaxed formulation to end up with a new problem whose numerical simulation leads to approximated optimal configurations. We include several such simulations in $2 \mathrm{~d}$ and $3 \mathrm{~d}$.
\end{abstract}

Mathematics Subject Classification. 49J45, 74P10, 74Q15.

Received June 2, 2004.

\section{INTRODUCTION}

We would like to review recent progress that has been made in the understanding and applicability of vector variational problems to several contexts in science and engineering. Our discussion is expository as we pretend to convey the main ideas without providing full proofs of facts which, on the other hand, are well-known and can be found in several places. Yet statements have been written down with care, and precise references are given.

We will start by briefly commenting on three situations to place our ideas in perspective, although we will particularly concentrate on optimal design problems. There are important different ingredients among those situations but they also share many common features. We would like to explore how a single, unified framework may help in treating all of them, emphasizing at the same time the relevance of their differences. All of those situations can be stated and considered under the form of the paradigmatic problem of the Calculus of Variations

$$
\text { Minimize in } u: \quad I(u)=\int_{\Omega} W(x, u(x), \nabla u(x)) \mathrm{d} x
$$

subject to appropriate further requirements on the competing vector fields $u$. The vector character is related to dimensions. We take $\Omega$ to be a regular, bounded domain in $\mathbf{R}^{N}$ (typically $N=2$ or 3 ), $u: \Omega \rightarrow \mathbf{R}^{m}$ has $m$ components so that $\nabla u$ is an $m \times N$ matrix. Finally

$$
W: \Omega \times \mathbf{R}^{m} \times \mathbf{M}^{m \times N} \rightarrow \mathbf{R}^{*}=\mathbf{R} \cup\{+\infty\}
$$

Keywords and phrases. Effective, homogenized or relaxed integrand, gradient Young measures, laminates.

1 ETSI Industriales, Universidad de Castilla-La Mancha, 13071 CiudadReal, Spain.

(C) EDP Sciences, SMAI 2005 
is the integrand characterizing our problem. We will identify this general problem as $(P)$. Specific properties (and obviously the particular form) of the integrand $W$ may lead to different ways of analyzing $(P)$.

If we classify variational problems like $(P)$ according to the criterium of how the value $+\infty$ is taken on by integrands, we may distinguish three types of problems:

1. The standard academic situation where

$$
W: \Omega \times \mathbf{R}^{m} \times \mathbf{M}^{m \times N} \rightarrow \mathbf{R}
$$

so that $W$ always takes on finite values, it is a Carathéodory function and even

$$
|A|^{p}-\frac{1}{c} \leq W(x, u, A) \leq C\left(|A|^{p}+1\right)
$$

for some $p>1$, and $0<c \leq C$. Several important variants can be considered depending on whether the exponents of the lower and upper bounds are different and what their relationship to dimensions is.

2. Nonlinear elasticity where $N=m=3$ or 2 ,

$$
W: \Omega \times \mathbf{R}^{m} \times \mathbf{M}^{m \times N} \rightarrow \mathbf{R}^{*}=\mathbf{R} \cup\{+\infty\}
$$

and the finite set for $W$ is

$$
\mathcal{F}_{W}=\left\{(x, u, A) \in \Omega \times \mathbf{R}^{N} \times M^{N \times N}: \operatorname{det} A>0\right\} .
$$

Yet $W$ is still a Carathéodory function in the sense that it is continuous on the pairs $(u, A)$ and hence the value $+\infty$ is taken on continuously. Even further the form of $W$ in nonlinear elasticity typically is

$$
W(x, u, A)=\tilde{W}(x, A)-F(x, u),
$$

for appropriate densities $\tilde{W}$ and $F$.

3. Optimal design where again $N=2$ or 3 but $m=j N$ with $j=1$ or 2 ,

$$
W: \Omega \times \mathbf{R}^{m} \times \mathbf{M}^{m \times N} \rightarrow \mathbf{R}^{*}=\mathbf{R} \cup\{+\infty\}
$$

and the finite set for $W$ is now a union of two (or more) manifolds in the space of matrices possibly depending on the other variables $(x, u)$. The interesting (and difficult) feature of $W$ is now that the value $+\infty$ is taken on abruptly. Still $W$ restricted to its finite set is a Carathéodory integrand. When we say that an integrand $W$ taking on thevalue $+\infty$ somewhere is a Carathéodory function, we mean that the restriction to its finite set is continuous on the $(u, A)$ variables and measurable on $x$.

These same considerations apply to integrands $V$ occurring inintegral constraints that must be respected, although it suffices to have the appropriate coercivity for one of the two integrands, either $W$ or $V$. When an integrand as in 2. or 3. above is said to be coercive, we mean that only the lower bound in (1.1) is required

$$
|A|^{p}-\frac{1}{c} \leq W(x, u, A)
$$

for $c>0, p>1$. Typically, $W$ will be coercive and then we only need $V$ to be bounded from below.

Since the first two cases are classical situations $[5,7,18,19]$, many of whose formal ingredients are rather well understood, we will dwell a bit on explaining in a typical, non-trivial but as simple as possible example, the third situation. This discussion is taken from [35].

Suppose we have at our disposal two conducting materials with conductivities $\alpha, \beta$ so that $0<\alpha<\beta$ and, given a design domain $\Omega \subset \mathbf{R}^{2}$, we would like to decide where to place the two materials so as to fill out all of $\Omega$ in such a way that a certain cost functional $J$ is minimized. $J$ will explicitly depend on the underlying 
electric field of the mixture of the two conducting materials; namely, if we solve the equilibrium equation of conductivity

$$
\begin{aligned}
-\operatorname{div}(\alpha \chi(x)+\beta(1-\chi(x)) \nabla u(x)) & =g \quad \text { in } \quad \Omega, \\
u & =u_{0} \quad \text { on } \quad \partial \Omega,
\end{aligned}
$$

then

$$
J(\chi)=\int_{\Omega}|\nabla u(x)|^{2} \mathrm{~d} x .
$$

Here the functions $g$ and $u_{0}$ are data of the problem and thus known. We could write down a much more general integrand for thecost functional depending on all the variables $(x, u, \nabla u, \chi)$ but since for this particular case our analysis can be carried out infull, we will restrict attention to this example. Notice that we have written $J(\chi)$ to emphasize that the characteristic function $\chi$ is the true design variable: it tells us where to put the $\alpha$-material. The amount of this material at our disposal is restricted so that

$$
\int_{\Omega} \chi(x) \mathrm{d} x \leq t_{0}|\Omega|
$$

and $t_{0} \in(0,1)$ is given. Altogether, we would like to

$$
\text { Minimize in } \chi: \quad J(\chi)=\int_{\Omega}|\nabla u(x)|^{2} \mathrm{~d} x
$$

subject to

$$
\begin{aligned}
-\operatorname{div}(\alpha \chi(x)+\beta(1-\chi(x)) \nabla u(x)) & =g \quad \text { in } \quad \Omega, \\
u & =u_{0} \quad \text { on } \quad \partial \Omega, \\
\int_{\Omega} \chi(x) \mathrm{d} x & \leq t_{0}|\Omega| .
\end{aligned}
$$

We can interpret this design problem as deciding where to place both materials so as to minimize the mean quadratic deviation from the zero vector field.

The reformulation of our problem starts with the realization that this equilibrium equation is equivalent (under the assumption of simple-connectedness of $\Omega$ ) to the existence of a potential (stream function) $v$ such that

$$
(\alpha \chi(x)+\beta(1-\chi(x))) \nabla u(x)+T \nabla v(x)+G(x)=0, \quad x \in \Omega,
$$

where $T$ is the $\pi / 2$, counter-clockwise rotation in the plane and $G$ is any vector field such that $\operatorname{div} G=g$. Recall that $\Omega \subset \mathbf{R}^{2}$. In this case we can alternatively use as design variables the pair of vector fields $(u, v)$ satisfying the additional, important, pointwise restriction

$$
\alpha \nabla u(x)+T \nabla v(x)+G(x)=0 \quad \text { or } \quad \beta \nabla u(x)+T \nabla v(x)+G(x)=0,
$$

for a.e. $x \in \Omega$. Notice how we can go from an admissible $\chi$ to such a pair $(u, v)$; and conversely, from a pair $(u, v)$ verifying (1.2) to an admissible $\chi$. Our aim now is to rewrite the optimal design problem in terms of these pairs $(u, v)$ instead of $\chi$.

To this end, collect both $u$ and $v$ in a single vector variable $U=\left(U^{(1)}, U^{(2)}\right)(u$ is identified with the first component $U^{(1)}$ while $v$ is identified with $\left.U^{(2)}\right)$. Define the two integrands

$$
W, V: M^{2 \times 2} \rightarrow \mathbf{R}^{*}=\mathbf{R} \cup\{+\infty\},
$$


by putting

$$
\begin{aligned}
& W(x, A)= \begin{cases}\left|A^{(1)}\right|^{2}, & \text { if } A \in \Lambda_{\alpha, x} \cup \Lambda_{\beta, x}, \\
+\infty, & \text { else, }\end{cases} \\
& V(x, A)= \begin{cases}1 /|\Omega|, & \text { if } A \in \Lambda_{\alpha, x}, \\
0, & \text { if } A \in \Lambda_{\beta, x}, \\
+\infty, & \text { else, }\end{cases} \\
& \Lambda_{\gamma, x}=\left\{A \in M^{2 \times 2}: \gamma A^{(1)}+T A^{(2)}+G(x)=0\right\}, \quad \gamma=\alpha \text { or } \beta .
\end{aligned}
$$

Then it is elementary to convince ourselves that the original optimal design problem is equivalent to the vector variational problem

$$
\text { Minimize in } U: \quad I(U)=\int_{\Omega} W(x, \nabla U(x)) \mathrm{d} x
$$

subject to

$$
U \in H^{1}(\Omega), \quad U^{(1)}=u_{0} \quad \text { on } \partial \Omega, \quad \int_{\Omega} V(x, \nabla U(x)) \mathrm{d} x \leq t_{0} .
$$

This final, explicit reformulation of the optimal design problem complies with the form of our model problem (P):

$$
\text { Minimize in } u \in \mathcal{A}: \quad I(u)=\int_{\Omega} W(x, u(x), \nabla u(x)) \mathrm{d} x,
$$

where the class of competing fields $\mathcal{A}$ is appropriately defined for each particular situation.

This sort of optimal design problems involving fine mixtures of the constituents have been addressed by homogenization techniques. See [1] for a nice, recent account on this. See also [40]. [13] is a reference for the more applied part of homogenization to engineering. In particular, the same problem we have described was proposed in [39] as a model problem to test homogenization techniques when cost functionals depend explicitly on $\nabla u$. [17] is an advanced book where a systematic treatment of unbounded functionals of the type we are here considering can be found.

Whenever we have to face a problem $(P)$ for a particular situation of the type described above, our main interest is to know the structure and features of some (ideally all) minimizing sequences including minimizers when they exist. Thisis a formal way of saying that we would like to know (all of) the optimal behavior for problem $(P)$. That is our goal, and all of our efforts are directed towards this aim. A generalapproach proceeds as follows.

We need to ensure, to begin with, that there are minimizing sequences for which the cost is finite and not arbitrarily close to $-\infty$. For that we assume that the functional $I(u)$ in problem $(P)$ is not identically $+\infty$ and thatthe integrand $W$ is bounded from below by some constant. Let $m$ stand for

$$
m=\inf \{I(u): u \in \mathcal{A}\} \in \mathbf{R},
$$

where $\mathcal{A}$ is, as indicated, the set of all admissible vector fields for $(P)$. $\mathcal{A}$ incorporates all kinds of restrictions to be respected by admissible $u$ 's including boundary conditions as well as integral constraints. We can therefore consider minimizing sequence $\left\{u_{j}\right\} \subset \mathcal{A}$ such that $I\left(u_{j}\right) \searrow m$. At this point we need some sort of compactness principle to detect a "limit object" from (possibly a subsequence of) $\left\{u_{j}\right\}$ so that we can say something about the relationship between $I\left(u_{j}\right)$ and its limit $m$ in terms of this new object. We know that when $\mathcal{A}$ is a subset of some Sobolev space, weak limits can be found under suitable uniform bounds in norm for the sequence $\left\{u_{j}\right\}$. However there is no a priori relationship between $I\left(u_{j}\right)$ and $I(u)$ because $\mathcal{A}$ could even fail to be weakly closed so that there is no guarantee that $u \in \mathcal{A}$. Young measures are tailored to be precisely these limit objects to describe the limit of (nonlinear) integral functionals. 
Theorem 1.1 [6]. Let $\Omega \subset \mathbf{R}^{N}$ be a measurable set andlet $z_{j}: \Omega \rightarrow \mathbf{R}^{m}$ be measurable functions such that

$$
\sup _{j} \int_{\Omega} h\left(\left|z_{j}\right|\right) \mathrm{d} x<\infty
$$

where $h:[0, \infty) \rightarrow[0, \infty]$ is a continuous,nondecreasing function such that $\lim _{t \rightarrow \infty} h(t)=\infty$. There exists a subsequence, not relabeled, and a family of probability measures, $\nu=\left\{\nu_{x}\right\}_{x \in \Omega}$ (the associated Young measure) depending measurably on $x$ with the property that whenever the sequence $\left\{\psi\left(x, z_{j}(x)\right)\right\}$ is weakly convergent in $L^{1}(\Omega)$ for any Carathéodory function $\psi(x, \lambda): \Omega \times \mathbf{R}^{m} \rightarrow \mathbf{R}^{*}$, the weak limit is the function

$$
\bar{\psi}(x)=\int_{\mathbf{R}^{m}} \psi(x, \lambda) \mathrm{d} \nu_{x}(\lambda) .
$$

The Young measure $\nu=\left\{\nu_{x}\right\}_{x \in \Omega}$ is generated by the sequence $\left\{z_{j}\right\}$ but it is independent of $\psi$.

Assume that we take $z_{j}(x)=\left(u_{j}(x), \nabla u_{j}(x)\right)$ where $\left\{u_{j}\right\}$ is minimizing for our problem $(P)$. If we wish to apply the above theorem to be able to extract a subsequence with an associated Young measure, we need the following coercivity on the integrand $W$

$$
h(|(u, A)|) \leq W(x, u, A)
$$

for a function $h$ as in Theorem 1.1. Then, possibly for a subsequence,

$$
\int_{\Omega} W\left(x, u_{j}(x), \nabla u_{j}(x)\right) \mathrm{d} x \rightarrow \int_{\Omega} \int_{\mathbf{R}^{m} \times \mathbf{M}^{m \times N}} W(x, \lambda, A) \mathrm{d} \nu_{x}(\lambda, A) \mathrm{d} x
$$

if $\nu=\left\{\nu_{x}\right\}_{x \in \Omega}$ is the Young measure associated with $\left\{z_{j}\right\}$. But it is valid provided that $\left\{W\left(x, u_{j}(x), \nabla u_{j}(x)\right)\right\}$ is weakly convergent in $L^{1}(\Omega)$. This may not be true at all. All we know is that it is bounded in $L^{1}(\Omega)$ because $\left\{u_{j}\right\}$ has been chosen to be minimizing. Concentration effects are responsible for non-convergent, bounded sequences in $L^{1}(\Omega)[9]$. Even so, the right inequality is always true. This whole discussion applies to $V$ as well, the integrand for integral constraints, as $V$ is bounded from below.

Theorem 1.2 [33]. If $\left\{z_{j}\right\}$ is a sequence of measurable functions withassociated Young measure $\nu=\left\{\nu_{x}\right\}_{x \in \Omega}$, then

$$
\liminf _{j \rightarrow \infty} \int_{E} \psi\left(x, z_{j}(x)\right) \mathrm{d} x \geq \int_{E} \int_{\mathbf{R}^{m}} \psi(x, \lambda) \mathrm{d} \nu_{x}(\lambda) \mathrm{d} x,
$$

for every Carathéodory function $\psi$, bounded from below, and every measurable subset $E \subset \Omega$.

In this way, we focus on Young measures associated with sequences $\left\{\left(u_{j}, \nabla u_{j}\right)\right\}$ of bounded sequences in Sobolev spaces. From now on, $\overline{\mathcal{A}}$ will designate the family of Young measures associated with such sequences when $u_{j} \in \mathcal{A}$. We then realize that it is important to move our main interest from problem $(P)$ to problem $(\bar{P})$ defined by

$$
\text { Minimize in } \nu \in \overline{\mathcal{A}}: \quad \bar{I}(\nu)=\int_{\Omega} \int_{\mathbf{R}^{m} \times \mathbf{M}^{m \times N}} W(x, \lambda, A) \mathrm{d} \nu_{x}(\lambda, A) \mathrm{d} x
$$

and $\overline{\mathcal{A}}$ includes all Young measures corresponding to all possible sequences $\left\{\left(u_{j}, \nabla u_{j}\right)\right\}$ where $u_{j} \in \mathcal{A}$. In particular, our main interest rests on those elements of $\overline{\mathcal{A}}$ generated by minimizing sequences of $(P)$. We suspect, based on the above representation formula of integral quantities in terms of Young measures, that these might be minimizers for $(\bar{P})$. So we are convinced that our main goal in studying $(P)$, that of understanding the structure of its minimizing sequences, may more easily be achieved by examining $(\bar{P})$ and hence we pursue the analysis of this new problem. But we face a tremendous difficulty as we do not have the slightest idea about what families of probability measures can enter into $\overline{\mathcal{A}}$ and, equally important, how to extract from a minimizer 
of $(\bar{P})$ a minimizing sequence of $(P)$. The rest of this presentation is an attempt to (partially) answer these two fundamental issues:

1. When does a given family of probability measures $\nu=\left\{\nu_{x}\right\}_{x \in \Omega}$ belong to $\overline{\mathcal{A}}$ ?

2. If $\nu=\left\{\nu_{x}\right\}_{x \in \Omega}$ does belong to $\overline{\mathcal{A}}$ and it is a minimizer for (1.3), how can one build a sequence of fields $u_{j} \in \mathcal{A}$ generating $\nu$ and minimizing for $(P)$ ?

We do not know how to solve these questions precisely and in full generality. All we have is some partial answers which in a number of situations lead to important, and even complete, developments. A first step in the understanding of $\overline{\mathcal{A}}$ consists in realizing that the important part of $\nu$ corresponding to $\left\{\left(u_{j}, \nabla u_{j}\right)\right\}$ is the one associated with the gradient part $\left\{\nabla u_{j}\right\}$.

Theorem 1.3 [33]. Let $z_{j}=\left(u_{j}, v_{j}\right): \Omega \rightarrow \mathbf{R}^{d} \times \mathbf{R}^{m}$ be a bounded sequence in $L^{p}(\Omega), p \geq 1$, such that $\left\{u_{j}\right\}$ converges strongly to $u$ in $L^{p}(\Omega)$. If $\nu=\left\{\nu_{x}\right\}_{x \in \Omega}$ is the Young measure associated with $\left\{z_{j}\right\}$, then $\nu_{x}=\delta_{u(x)} \otimes \mu_{x}$ a.e. $x \in \Omega$, where $\left\{\mu_{x}\right\}_{x \in \Omega}$ is the Young measure corresponding to $\left\{v_{j}\right\}$.

Because of this fact, we will identify henceforth $\overline{\mathcal{A}}$ with the class of Young measures corresponding to sequences $\left\{\nabla u_{j}\right\}$ for $u_{j} \in \mathcal{A}$ and assume the coercivity

$$
|A|^{p}-\frac{1}{c} \leq W(x, \lambda, A)
$$

$c>0, p>1$, for all $(x, \lambda)$.

Assume we have two classes of families of probability measures such that

$$
\overline{\mathcal{A}}_{*} \subset \overline{\mathcal{A}} \subset \overline{\mathcal{A}}^{*} .
$$

Suppose we know how to explicitly determine $\overline{\mathcal{A}}^{*}$, without any reference to $\mathcal{A}$ or to gradients, and how to build generating sequences $\left\{u_{j}\right\}$ for the families of probability measures in $\overline{\mathcal{A}}_{*}$ so that $u_{j} \in \mathcal{A}$ and $I\left(u_{j}\right) \rightarrow \bar{I}(\nu)$, $\nu \in \overline{\mathcal{A}}_{*}$. If the problem of deciding whether these different families, $\overline{\mathcal{A}}_{*}$ and $\overline{\mathcal{A}}^{*}$, are in fact the same is out of reach (this is indeed the case), there is still something we can do about the analysis of $(\bar{P})$. Consider a new, generalized variational problem, which we will designate $\left(\bar{P}^{*}\right)$,

$$
\text { Minimize in } \nu \in \overline{\mathcal{A}}^{*}: \quad \bar{I}(\nu) \text {. }
$$

Suppose $\nu_{0}$ is a minimizer for $\left(\bar{P}^{*}\right)$, which may be relatively easy to find if we have a clear way of defining elements in $\overline{\mathcal{A}}^{*}$ as indicated earlier. Suppose we are so lucky that such minimizer $\nu_{0}$ turns out to belong to $\overline{\mathcal{A}}_{*}$. Then we know how to construct generating sequences for $\nu_{0}$ and those will be minimizing for $(P)$ thus providing some optimal behavior for our initial optimization problem $(P)$. If this is so, we will have (partially) succeded in achieving our goal. Indeed, notice that if $\bar{m}^{*}$ is the minimum for $\left(\bar{P}^{*}\right)$ and $\nu_{0} \in \overline{\mathcal{A}}^{*} \cap \overline{\mathcal{A}}_{*}$ is a minimizer for this problem, then

$$
\bar{I}\left(\nu_{0}\right)=\bar{m}^{*} \leq \bar{m} \leq m,
$$

if $\bar{m}$ and $m$ are the minima (infima) for $(\bar{P})$ and $(P)$, respectively. On the other hand, because $\nu_{0} \in \overline{\mathcal{A}}_{*}$ and we are assuming that we know how to build a sequence $\left\{u_{j}\right\} \subset \mathcal{A}$ such that

$$
I\left(u_{j}\right) \searrow \bar{I}\left(\nu_{0}\right),
$$

we will have that in fact

$$
I\left(u_{j}\right) \searrow m
$$

and $\left\{u_{j}\right\}$ is a minimizing sequence. Finding such a minimizing sequence is our goal.

The aim of these pages is two-fold. On the one hand, to provide a more precise overview of the philosophy delineated on the preceding paragraphs. On the other, to emphasize the peculiarities of such approach associated 
with the third situation described earlier in which integrands take on the value $+\infty$ abruptly. Special attention will be dedicated to optimal design problems and their variational reformulation, as we would like to show how all this programme can and has been carried out in some interesting situations including the one described earlier in two-dimensional conductivity. The typical academic problem has been the subject of much attention since the pioneering work of Morrey [31,32]. See also [19,33]. Nonlinear elasticity has also received a lot of attention $[2,5,8,18,34]$. In Section 2 we will explain the new families of measures $\overline{\mathcal{A}}_{*}$ and $\overline{\mathcal{A}}^{*}$ in the context of the optimal design problem described earlier, as well as how generating sequences are constructed for families of measures in $\overline{\mathcal{A}}_{*}$ and how elements in $\overline{\mathcal{A}}^{*}$ are defined, respectively. We will also reserve some words for $\overline{\mathcal{A}}$. We will then describe the strategy to solve $\left(\bar{P}^{*}\right)$, and proceed to explain how minimizing sequences and optimal distributions of materials for the initial optimal design problem can be found (Sect. 5).

\section{The Classes $\overline{\mathcal{A}}^{*}$ And $\overline{\mathcal{A}}_{*}$}

We would like to define $\overline{\mathcal{A}}^{*}$ by keeping some of the fundamental restrictions that a Young measure generated by gradients should verify. Emphasis is placed on the gradient requirement as we know that this is not irrelevant and must play a fundamental role. Our discussion will not mention many terms closely related to this subject like weak lower semicontinuity, polyconvexity, characterization of gradient Young measures, Jensen's inequality, etc. $[4,19,27,33]$.

The main feature of Young measures generated by gradients is contained in the following theorem. It is a well-known result that establishes that subdeterminants commute with integration against a Young measure generated by gradients.

Theorem 2.1. Let $\nu=\left\{\nu_{x}\right\}_{x \in \Omega}$ be generated by $\left\{\nabla u_{j}\right\}$ where $\left\{u_{j}\right\}$ is a bounded sequence in $W^{1, p}(\Omega)$. If $r \leq p$, then for any minor $M$ of order $r$ we have

$$
M\left(\int_{\mathbf{M}^{m \times N}} A \mathrm{~d} \nu_{x}(A)\right)=\int_{\mathbf{M}^{m \times N}} M(A) \mathrm{d} \nu_{x}(A) .
$$

This theorem itself is a direct consequence of a fundamental fact about weak continuity for minors $[5,38]$.

Lemma 2.2. If $u_{j} \rightarrow u$ in $W^{1, p}(\Omega)$, then $M\left(\nabla u_{j}\right) \rightarrow M(\nabla u)$ in the sense of distributions whenever $r \leq p$ and $r$ is the order of the minor (subdeterminant) $M$.

This weak convergence in the sense of distributions can be improved to weak convergence (with the same limit $M(\nabla u))$ whenever the sequence $\left\{M\left(\nabla u_{j}\right)\right\}$ does converge in the weak sense. In this case, if we interpret this weak continuity result in terms of associated Young measures we immediately get Theorem 2.1.

Finally, it simplifies the argument if we may rely on a localization result so that all of our assertions on the classes of measures can be done in a pointwise fashion.

Lemma 2.3 [33]. Let $\nu=\left\{\nu_{x}\right\}_{x \in \Omega}$ be a Young measure generated by a bounded sequence of gradients in $W^{1, p}(\Omega)$. For a.e. $a \in \Omega$ and for any domain $Q$, there exists a bounded sequence in $W^{1, p}(Q),\left\{v_{a, j}\right\}$, such that the Young measure associated with $\left\{\nabla v_{a, j}\right\}$ is $\nu_{a}$, homogeneous (the same for all points in $Q$ ). Moreover each function $v_{a, j}$ can be chosen in such a way that $v_{a, j}-u_{F(a)} \in W_{0}^{1, p}(Q)$, where $u_{F}(x)$ is the linear function $F x$ for $F \in \mathbf{M}^{m \times N}, x \in Q$, and

$$
F(a)=\int_{\mathbf{M}} A \mathrm{~d} \nu_{a}(A)
$$

We can now explicitly define $\overline{\mathcal{A}}^{*}$ as the set of probability measures whose first moment is the gradient of a function (that may not belong to $\mathcal{A}$ ) and commute with all (or some) subdeterminants. Notice that we are defining $\overline{\mathcal{A}}^{*}$ by retaining some of the properties we know that Young measures generated by gradients should 
satisfy. Our hope is to have thus kept some of the key features of those families of probability measures. More explicitly we can say that $\overline{\mathcal{A}}^{*}$ is defined by

1. polynomial growth:

$$
\int_{\Omega} \int_{\mathbf{M}^{m \times N}}|A|^{p} \mathrm{~d} \nu_{x}(A) \mathrm{d} x<+\infty, \quad p \geq \min \{m, N\}
$$

2. compatibility throughout $\Omega$ : there exists $u \in W^{1, p}(\Omega)$ such that

$$
\int_{\mathbf{M}^{m \times N}} A \mathrm{~d} \nu_{x}(A)=\nabla u(x) \text { for a.e. } x \in \Omega
$$

3. commutation with weak continuous functions: for all (or some) minors $M$ and a.e. $x \in \Omega$

$$
M\left(\int_{\mathbf{M}^{m \times N}} A \mathrm{~d} \nu_{x}(A)\right)=\int_{\mathbf{M}^{m \times N}} M(A) \mathrm{d} \nu_{x}(A) ;
$$

4. integral constraints if present:

$$
\int_{\mathbf{M}^{m \times N}} V(x, A) \mathrm{d} \nu_{x}(A)=t(x), \quad \int_{\Omega} t(x) \mathrm{d} x \leq t_{0} .
$$

The first requirement above comes from the coercivity Condition (1.4) for such exponent $p$ while the last condition corresponds to the volume integral constraint.

We now turn to defining the other class $\overline{\mathcal{A}}_{*}$. This material is taken from [33]. To this aim, we recall a basic but important construction. Let $F_{i} \in \mathbf{M}^{m \times N}, i=1,2, a \in \mathbf{R}^{m}$ and a unit vector $n \in \mathbf{R}^{N}$ be given in such a way that

$$
F_{1}-F_{2}=a \otimes n
$$

If $\chi_{t}$ is the characteristic function of the interval $(0, t)$ in $(0,1)$ extended by periodicity, the Young measure associated with the sequence of gradients

$$
\begin{gathered}
\nabla u_{j}(x)=F_{2}+\chi_{t}(j x \cdot n) a \otimes n, \\
u_{j}(x)=F_{2} x+\frac{1}{j} \int_{0}^{j x \cdot n} \chi_{t}(s) \mathrm{d} s a,
\end{gathered}
$$

is

$$
\nu_{x}=\nu=t \delta_{F_{1}}+(1-t) \delta_{F_{2}}, \quad x \in \Omega,
$$

where $\Omega$ is any bounded domain in $\mathbf{R}^{N}$ (Fig. 2.1). Therefore the probability measure $\nu$ in (2.2) is a gradient Young measure (generated by a sequence of gradients) for any $t \in[0,1]$ provided the compatibility Condition (2.1) holds.

We can assume without loss of generality (this involves a basic, elementary fact about cut-off functions) that $u_{j}-u_{F} \in W_{0}^{1, \infty}(\Omega), F=t F_{1}+(1-t) F_{2}$ and $u_{F}(x)=F x$. Moreover $\nabla u_{j}$ takes on thevalues $F_{1}$ and $F_{2}$ except in small sets $E_{j},\left|E_{j}\right| \rightarrow 0$. We would like to go one step further in this construction. Assume, in addition to $(2.1)$, that

$$
\begin{gathered}
F_{2}=t_{0} F_{2}^{(1)}+\left(1-t_{0}\right) F_{2}^{(2)}, \quad t_{0} \in(0,1), \\
F_{2}^{(1)}-F_{2}^{(2)}=b \otimes e,
\end{gathered}
$$

where $b \in \mathbf{R}^{m}$, and $e \in \mathbf{R}^{N}$ is another unit vector. Let $\Omega_{i}^{j}$ be the part of $\Omega$ where $\nabla u_{j}=F_{i}, i=1,2$. For $j$ and $i$ fixed, based on the compatibility condition between $F_{2}^{(1)}$ and $F_{2}^{(2)}$, we can construct a sequence of gradients $\left\{\nabla v_{k}^{j i}\right\}, v_{k}^{j i}-u_{F_{2}} \in W_{0}^{1, \infty}\left(\Omega_{i}^{j}\right)$, whose values essentially alternate between $F_{2}^{(1)}$ and $F_{2}^{(2)}$ with preassigned 


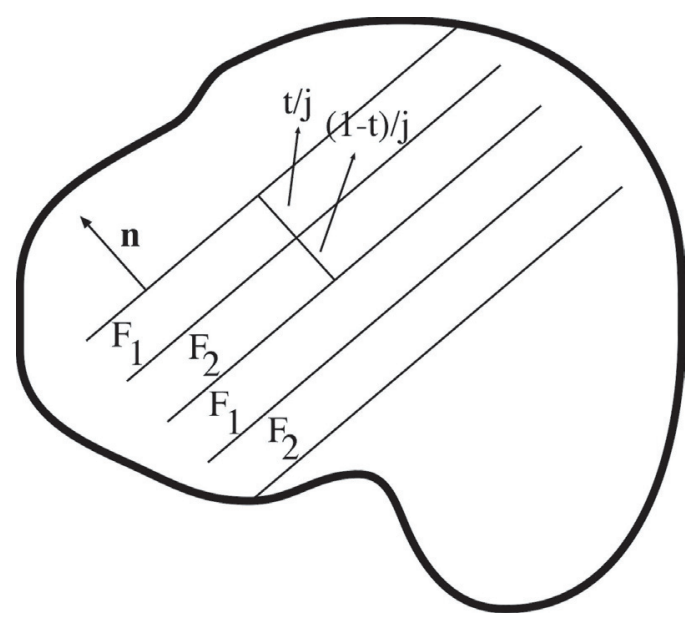

Figure 2.1. A first-order laminate.

frequency $t_{0} \in(0,1)$ and normal $e$ to the layers. Let $E_{k}^{j i}$ be the set where $\nabla v_{k}^{j i}$ does not take either of the two values $F_{2}^{(1)}$ or $F_{2}^{(2)}$. Choose $k=k(j, i)$ in such a way that

$$
\frac{\left|E_{k}^{j i}\right|}{\left|\Omega_{i}^{j}\right|} \rightarrow 0
$$

as $j \rightarrow \infty$ uniformly in $i=1,2$. Define

$$
u^{(j)}(x)= \begin{cases}v_{k(j, i)}^{j i}(x), & x \in \Omega_{i}^{j}, \\ u_{j}(x), & \text { else. }\end{cases}
$$

This sequence $\left\{u^{(j)}\right\}$ is uniformly bounded in $W^{1, \infty}(\Omega)$ and satisfies $u^{(j)}-u_{F} \in W_{0}^{1, \infty}(\Omega)$. The Young measure associated with $\left\{\nabla u^{(j)}\right\}$ is

$$
\nu=t \delta_{F_{1}}+(1-t)\left(t_{0} \delta_{F_{2}^{(1)}}+\left(1-t_{0}\right) \delta_{F_{2}^{(2)}}\right)
$$

homogeneous, the same for all points in the domain. The probability measure in (2.4) is a gradient Young measure provided we have the compatibility Conditions (2.1) and (2.3). See Figure 2.2 for the structure of those gradients.

It is not difficult to generalize this construction when a finite number of matrices is involved if we have the rank-one condition satisfied in a recursive way. This basic construction has been referred to as "layers within layers" in the literature and reflects intuitively the situation. It motivates the following definition.

Definition 2.4 [19]. A set of pairs $\left\{\left(t_{i}, Y_{i}\right)\right\}_{1 \leq i \leq l}$ where $t_{i}>0, \sum_{i} t_{i}=1, Y_{i} \in \mathbf{M}^{m \times N}$ is said to satisfy the $\left(H_{l}\right)$ condition if:

i) for $l=2, \operatorname{rank}\left(Y_{1}-Y_{2}\right) \leq 1$;

ii) if $l>2$ and possibly after a permutationof indices, $\operatorname{rank}\left(Y_{1}-Y_{2}\right) \leq 1$ and if we set

$$
\begin{gathered}
s_{1}=t_{1}+t_{2}, \quad Z_{1}=\frac{t_{1}}{s_{1}} Y_{1}+\frac{t_{2}}{s_{1}} Y_{2}, \\
s_{i}=t_{i+1}, \quad Z_{i}=Y_{i+1}, \quad 2 \leq i \leq l-1,
\end{gathered}
$$

then the set of pairs $\left\{\left(s_{i}, Z_{i}\right)\right\}_{1 \leq i \leq l-1}$ satisfies the $\left(H_{l-1}\right)$ condition. 


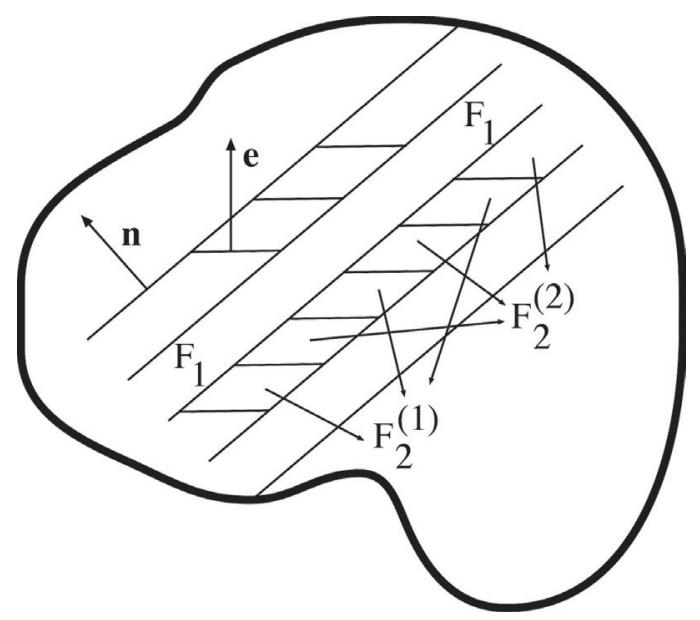

Figure 2.2. A second-order laminate.

An immediate consequence of our previous discussion is contained in the next statement.

Proposition 2.5. If $\left\{\left(t_{i}, Y_{i}\right)\right\}_{1 \leq i \leq l}$ satisfies the $\left(H_{l}\right)$ condition, then the probability measure $\nu=\sum_{i} t_{i} \delta_{Y_{i}}$ is a gradient Young measure.

We can even take weak $*$ limits in the sense of measures for sequences of finite convex combinations of Dirac masses verifying $\left(H_{l}\right)$ conditions. These weak limits will also be gradient Young measures: the argument iselementary and involves taking diagonal sequences. Notice that in fact the set of homogeneous gradient Young measures is weak * closed. This remark motivates the definition of laminates. For those readers not familiar with the notion of weak $*$ convergence of measures, these weak $*$ limits can be interpreted as $\left(H_{l}\right)$ conditions when $l \rightarrow \infty$. In this case we talk about infinite order laminates.

Definition 2.6. Let $\nu$ be a probability measure supported on $\mathbf{M}^{m \times N}$ and let $K=\operatorname{supp}(\nu)$ be a compact set. $\nu$ is a laminate if there exists a sequence of sets of pairs $\left\{\left(t_{i}^{k}, Y_{i}^{k}\right)\right\}_{1 \leq i \leq k},(k \geq 2)$, verifying the $\left(H_{k}\right)$ condition such that $\sum_{i} t_{i}^{k} \delta_{Y_{i}^{k}} \stackrel{*}{\rightarrow} \nu$ in the sense of measures.

Proposition 2.7. Every laminate is a (homogeneous) gradient Young measure.

We see that the essential ingredient to build laminates is expressed in the $\left(H_{l}\right)$ condition, and the recursive rank-one compatibility is the key requirement. A patching procedure allows us to build a global Young measure generated by gradients from laminates at every point of the domain.

Lemma 2.8 [27]. Let $\nu=\left\{\nu_{x}\right\}_{x \in \Omega}$ be a family of probability measures verifying the following requirements:

1. the first moment is a gradient

$$
\nabla u(x)=\int_{\mathbf{M}^{m \times N}} A \mathrm{~d} \nu_{x}(A), \quad u \in W^{1, p}(\Omega) ;
$$

2. every member of the family $\nu_{x}$, for a.e. fixed $x \in \Omega$, can be generated by a sequence of gradients (in particular if each $\nu_{x}$ is a laminate);

3. integrability of the $p$-th power

$$
\int_{\Omega} \int_{\mathbf{M}^{m \times N}}|A|^{p} \mathrm{~d} \nu_{x}(A) \mathrm{d} x<+\infty .
$$


Then there exists a bounded sequence $\left\{u_{j}\right\}$ in $W^{1, p}(\Omega)$ such that $\nu=\left\{\nu_{x}\right\}_{x \in \Omega}$ is generated by $\left\{\nabla u_{j}\right\}$. In addition, the sequence $\left\{u_{j}\right\}$ can be chosen so that $\left\{\left|\nabla u_{j}\right|^{p}\right\}$ is equiintegrable in $\Omega$.

When integrands behave like the $p$-th power at infinity, this patching procedure is a general result that allows us to care just about the gradient requirement locally around each $x \in \Omega$. However, this is not so in general when integrands take on infinite values as generating sequences of gradients must always take values on the finite set of integrands. It is not enough that this happens for small sets. This is in fact an important property of sets of matrices. Given a certain set of matrices $\Lambda$ (e.g. the finite set for an integrand), and a gradient Young measure $\nu$ supported in such set, one interesting issue is whether one can find a generating sequence of gradients $\left\{\nabla u_{j}\right\}$ supported on such set

$$
\left|\left\{\nabla u_{j} \in \Lambda\right\}\right|=|\Omega| \text { for all } j
$$

under additional restrictions related, for instance, to boundary values and/or integral constraints. It does not suffice just to have

$$
\left|\left\{\nabla u_{j} \in \Lambda\right\}\right| \rightarrow|\Omega| \quad \text { as } j \rightarrow \infty
$$

as the smallness of the sets $\left\{\nabla u_{j} \notin \Lambda\right\}$ might be compensated with high values of the integrand $W$ over them to yield a non-negligible contribution to the integral cost functional. Each such situation should be treated carefully and separately. See [24].

We define $\overline{\mathcal{A}}_{*}$ as the set of probability measures whose first moment is the gradient of a function in $W^{1, p}(\Omega)$, each individual member for a.e. $x \in \Omega$ is a laminate and we have the appropriate integrability as stated in Lemma 2.8. By our discussion, this is a subclass of the family of Young measures that can be generated by gradients. Notice that in the situation of our optimal design problem $\overline{\mathcal{A}}_{*} \subset \overline{\mathcal{A}}^{*}$ because $\overline{\mathcal{A}}$ is a subset of the latter (gradient Young measures belong to $\overline{\mathcal{A}}^{*}$ ) while it contains the former (laminates are gradient Young measures). This issue can be much more delicate in other contexts.

Our general strategy is to study the generalized variational problem $\left(\bar{P}^{*}\right)$ defined over $\overline{\mathcal{A}}^{*}$, and hope that we can find at least one optimal family of probability measures that is a laminate at every point satisfying all additional requirements we ought to meet so that, through the patching procedure Lemma 2.8 , we can build a minimizing sequence for our original optimization problem $(P)$ which is, after all, our main goal as stated in the Introduction.

We then proceed to examine $\left(\bar{P}^{*}\right)$.

\section{Generalized Minimizers}

In this section we would like to describe the main ideas in exploring the variational problem $\left(\bar{P}^{*}\right)$

$$
\text { Minimize in } \nu \in \overline{\mathcal{A}}^{*}: \quad \bar{I}(\nu) \text {. }
$$

For simplicity, although more general situations can also be handled, we will assume as in our model problem in the Introduction that

$$
W, V: \Omega \times \mathbf{M}^{m \times N} \rightarrow \mathbf{R}^{*}
$$

so that we will drop the explicit dependence on the variable $u$. Recall that $\overline{\mathcal{A}}^{*}$ is the class of families of probability measures $\nu=\left\{\nu_{x}\right\}_{x \in \Omega}$ verifying these requirements:

1. polynomial growth:

$$
\int_{\Omega} \int_{\mathbf{M}^{m \times N}}|A|^{p} \mathrm{~d} \nu_{x}(A) \mathrm{d} x<+\infty, \quad p \geq \min \{m, N\} ;
$$

2. compatibility throughout $\Omega$ : there exists $u \in W^{1, p}(\Omega)$ such that

$$
\int_{\mathbf{M}^{m \times N}} A \mathrm{~d} \nu_{x}(A)=\nabla u(x) \quad \text { for a.e. } x \in \Omega
$$


3. commutation with weak continuous functions: for all (or some) minors $M$ and a.e. $x \in \Omega$

$$
M\left(\int_{\mathbf{M}^{m \times N}} A \mathrm{~d} \nu_{x}(A)\right)=\int_{\mathbf{M}^{m \times N}} M(A) \mathrm{d} \nu_{x}(A) ;
$$

4. integral constraints if present:

$$
\int_{\mathbf{M}^{m \times N}} V(x, A) \mathrm{d} \nu_{x}(A)=t(x), \quad \int_{\Omega} t(x) \mathrm{d} x \leq t_{0} .
$$

Once we have very clearly stated the properties of competing families of probability measures, the next step is to factor out the minimization process in (3.1) through their first moment, which is the gradient of a vector field in $W^{1, p}(\Omega)$, and the local integral constraint

$$
t(x)=\int_{\mathbf{M}^{m \times N}} V(x, A) \mathrm{d} \nu_{x}(A), \quad \int_{\Omega} t(x) \mathrm{d} x \leq t_{0} .
$$

If we let $\mathcal{A}^{(1)}$ stand for the set of pairs $(u, t)$ such that (3.2) and (3.3) hold for some $\nu \in \overline{\mathcal{A}}^{*}$, then we write $\inf \left\{\bar{I}(\nu): \nu \in \overline{\mathcal{A}}^{*}\right\}=$

$$
\inf _{(u, t) \in \mathcal{A}^{(1)}}\left\{\inf \left\{\bar{I}(\nu): \nu \in \overline{\mathcal{A}}^{*}, \nabla u(x)=\int_{\mathbf{M}^{m \times N}} A \mathrm{~d} \nu_{x}(A), t(x)=\int_{\mathbf{M}^{m \times N}} V(x, A) \mathrm{d} \nu_{x}(A)\right\}\right\} .
$$

More clearly, if for fixed $(u, t) \in \mathcal{A}^{(1)}$ we denote

$$
\tilde{I}(u, t)=\inf \left\{\bar{I}(\nu): \nu \in \overline{\mathcal{A}}^{*}, \nabla u(x)=\int_{\mathbf{M}^{m \times N}} A \mathrm{~d} \nu_{x}(A), t(x)=\int_{\mathbf{M}^{m \times N}} V(x, A) \mathrm{d} \nu_{x}(A)\right\},
$$

then

$$
\inf \left\{\bar{I}(\nu): \nu \in \overline{\mathcal{A}}^{*}\right\}=\inf \left\{\tilde{I}(u, t):(u, t) \in \mathcal{A}^{(1)}\right\} .
$$

It is interesting to point out that the functional $\tilde{I}(u, t)$ is in fact local. This is a consequence of a more general version of Lemma 2.3 that takes also into account the $t$-dependence (see [25] for a similar situation). It admits therefore an integral representation of the type

$$
\tilde{I}(u, t)=\int_{\Omega} C P W(x, \nabla u(x), t(x)) \mathrm{d} x
$$

where the integrand is defined by

$$
C P W(x, A, t)=\inf \left\{\int_{\mathbf{M}^{m \times N}} W(x, A) \mathrm{d} \mu(A): \mu \in \overline{\mathcal{A}}_{0}^{*}(x, A, t)\right\}
$$

and $\overline{\mathcal{A}}_{0}^{*}(x, A, t)$ designates the class of probability measures commuting with the chosen minors $M$ in the definition of $\overline{\mathcal{A}}^{*}$, having barycenter $A$ and integral against $V(x, \cdot)$ equal to $t$. The notation $C P W$ has been chosen because this integrand is known as the (constrained) polyconvexification of $W$. When the same scheme is followed for the class $\overline{\mathcal{A}}$ instead of $\overline{\mathcal{A}}^{*}$ the integrand that one obtains, $C Q W$, is called the (constrained) quasiconvexification of $W$ and the fact that the infima of $I$ and of $\tilde{I}$ over the same class $\mathcal{A}$ coincide, is known as a relaxation theorem $[12,19,25]$. 
We now comment on the outer minimization problem

$$
\inf \left\{\tilde{I}(u, t):(u, t) \in \mathcal{A}^{(1)}\right\}=\inf \left\{\int_{\Omega} C P W(x, \nabla u(x), t(x)) \mathrm{d} x:(u, t) \in \mathcal{A}^{(1)}\right\} .
$$

This is a variational problem that looks like the original one we started with only with a different integrand and a different set of competing functions. The explicit computation of $\tilde{I}(u, t)$ should deliver a clear definition of $\mathcal{A}^{(1)}$.

This new variational problem is however very different from the original one unless $C P W$ turns out to be exactly $W$ and no integral constraint is to be respected. This will be so if the initial integrand $W$ was polyconvex from the beginning. Our main result in this section establishes that all infima ocurring above are in fact minima under coercivity assumptions on $W[3]$.

Theorem 3.1. Suppose $W$ and $V$ are Carathéodory functions as indicated earlier with

$$
|A|^{p}-\frac{1}{c} \leq W(x, A)
$$

where $c>0, p \geq \min \{m, N\}$, and $V$ is bounded from below.

1. Whenever $\overline{\mathcal{A}}_{0}^{*}(x, A, t)$ is non-empty, the infimum in (3.4) is attained (it is a minimum).

2. The variational problem (3.5) admits optimal solutions.

We are now ready to describe how this whole discussion supports the following procedure when trying to solve our original variational problem which means to detect the structure of some minimizing sequences.

1. Solve explicitly, when possible, the problem of the (constrained) polyconvexification

$$
C P W(x, A, t)=\min \left\{\int_{\mathbf{M}^{m \times N}} W(x, A) \mathrm{d} \mu(A): \mu \in \overline{\mathcal{A}}_{0}^{*}(x, A, t)\right\} .
$$

This requires to obtain the value $C P W(x, A, t)$ as well as a measure minimizer, $\mu_{x, A, t}$, in $\overline{\mathcal{A}}_{0}^{*}(x, A, t)$ so that

$$
C P W(x, A, t)=\int_{\mathbf{M}^{m \times N}} W(x, F) \mathrm{d} \mu_{x, A, t}(F) .
$$

2. Determine the optimal pair, $(U, T)$, of the problem

$$
\text { Minimize in }(u, t) \in \mathcal{A}^{(1)}: \quad \int_{\Omega} C P W(x, \nabla u(x), t(x)) \mathrm{d} x .
$$

Notice that $\mathcal{A}^{(1)}$ is the class of pairs $(u, t)$ forwhich $\tilde{I}(u, t)$ is finite. To deal with this step, we can use the informationcoming from optimality conditions. Notice that this will be a system of PDE possibly incorporating multipliers. We could alternatively try to directly approximate the minimizer or simplify the problem in some other way.

3. For the optimal solution $(U, T)$ of this regular variational problem, go back to the constrained polyconvexification and check whether the optimal measure $\mu_{x, \nabla U(x), T(x)}$ is a laminate for a.e. $x \in \Omega$ and if a sequence $\left\{u_{j}\right\} \subset \mathcal{A}$ can be generated taking values on the finite set of $W$ at every point in $\Omega$. Such sequence is the target of all of our analysis as it will be minimizing for our original problem $(P)$.

If the last step can be carried out, we have succeded in our endeavor. If not, we may have much information but not quite what we were looking for. In fact, little can be done if this whole process fails. In some instances the optimal measures $\mu_{x, A, t}$ found in the first step above are already laminates for all such pairs regardless of the particular $x$-dependence.

For problems where optimal polyconvex measures are expected to be as simple as possible in the sense that their support is just a few mass points, there is a great deal of hope that the process described will succeed. 
Indeed, the optimality requirement on these measures providing the polyconvexification has a lot to do with their support being as small as possible. Sometimes it may be impossible to find the optimal solution $U$. In many instances, all we are looking for is some extra information so that we can succeed in the final step of showing that optimal polyconvex measures are indeed laminates all over the domain. In this case, one can often setup a simpler variational problem encoding the behavior of minimizing sequences which can be approximated numerically in a suitable way. Typically, the main step of this whole programme reduces to the more-or-less explicit form of the constrained polyconvexification especially when some of the optimal measures turn out to be laminates. When this is the case we end up with a new, equivalent variational problem which admits optimal solutions. These optimal solutions together with the associated optimal measures encode all the ingredients to reconstruct minimizing sequences for the original optimization problem.

This philosophy of retaining some of the key ingredients of gradient Young measures in an attempt to compute quasiconvex hulls is the only known way to do so. It has been used in a number of interesting situations where it has led to successful results. See some important examples in $[10,14-16,20,28,29]$.

We would like to treat the optimal design problem described earlier to show how this whole programme can be applied and in some cases provide theclue to optimal behavior.

\section{Optimal Design}

We will describe one of the simplest situations where the approach described above can be carried out in full:

$$
\text { Minimize in } \chi: \quad I(\chi)=\int_{\Omega}|\nabla u(x)|^{2} \mathrm{~d} x
$$

subject to

$$
\begin{aligned}
-\operatorname{div}(\alpha \chi(x)+\beta(1-\chi(x)) \nabla u(x)) & =\operatorname{div} G \quad \text { in } \quad \Omega, \\
u & =u_{0} \quad \text { on } \partial \Omega, \\
\int_{\Omega} \chi(x) \mathrm{d} x & \leq|\Omega| t_{0},
\end{aligned}
$$

where $\Omega, \varphi, G, u_{0}, 0<\alpha<\beta, 0<t_{0}<1$ are the data set of theproblem. The cost functional can more generally be taken as

$$
I(\chi)=\int_{\Omega}|\nabla u(x)-P(x)|^{2} \mathrm{~d} x
$$

for an arbitrary vector field $P$ in $L^{2}(\Omega)$, but we will retain $P \equiv 0$ for simplicity. Note that both dimensions $m$ and $N$ are two so that we will work with the space of $2 \times 2$ matrices. This was proposed as a test problem in [39] to examine how the ideas of homogenization could be pushed to treat an explicit dependence of the cost functional on the field. It was later retaken in [26] and [30].

Let us recall the equivalent vector variational problem

$$
\text { Minimize in } U: \quad \tilde{I}(U)=\int_{\Omega} W(x, \nabla U(x)) \mathrm{d} x
$$

subject to

$$
U \in H^{1}(\Omega), \quad U^{(1)}-u_{0} \in H_{0}^{1}(\Omega), \quad \int_{\Omega} V(x, \nabla U(x)) \mathrm{d} x \leq t_{0} .
$$

The integrands for this variational problem are

$$
W(x, A)= \begin{cases}\left|A^{(1)}\right|^{2}, & \text { if } A \in \Lambda_{\alpha, x} \cup \Lambda_{\beta, x}, \\ +\infty, & \text { else, }\end{cases}
$$


where

$$
\Lambda_{\gamma, x}=\left\{A \in M^{2 \times 2}: \gamma A^{(1)}+T A^{(2)}+G(x)=0\right\} .
$$

The integral constraint can be expressed through the integrand

$$
V(x, A)= \begin{cases}1 /|\Omega|, & \text { if } A \in \Lambda_{\alpha, x} \\ 0, & \text { if } A \in \Lambda_{\beta, x} \backslash \Lambda_{\alpha, x} \\ +\infty, & \text { else }\end{cases}
$$

The main step of our programme is the computation of the constrained polyconvexification

$C P W(x, F, t)=\min \left\{\int_{M^{2 \times 2}} W(x, A) \mathrm{d} \nu(A): \nu\right.$ commutes with $\operatorname{det}$,

$$
\left.\int_{M^{2 \times 2}} A \mathrm{~d} \nu(A)=F, \int_{M^{2 \times 2}} V(x, A) \mathrm{d} \nu(A)=t\right\} .
$$

The discussion that follows is essentially taken from $[35,36]$. Since the spatial variable $x \in \Omega$ can be regarded as a parameter, and it is irrelevant from the point of the computation of $C P W$, to simplify notation we will drop this dependence by assuming $G \equiv 0$ in the arguments that follow. For a non-vanishing $G$, the formula for $C P W$ is obtained by an appropriate translation. Thus we will take

$$
\begin{aligned}
& W(A)= \begin{cases}\left|A^{(1)}\right|^{2}, & \text { if } A \in \Lambda_{\alpha} \cup \Lambda_{\beta}, \\
+\infty, & \text { else, }\end{cases} \\
& \Lambda_{\gamma}=\left\{A \in M^{2 \times 2}: \gamma A^{(1)}+T A^{(2)}=0\right\}, \\
& V(A)= \begin{cases}1 /|\Omega|, & \text { if } A \in \Lambda_{\alpha}, \\
0, & \text { if } A \in \Lambda_{\beta} \backslash \Lambda_{\alpha}, \\
+\infty, & \text { else. }\end{cases}
\end{aligned}
$$

In this situation $C P W$ does not depend on $x$ and for each pair $(F, t), C P W(F, t)$ is computed by solving the optimization problem

subject to

$$
\text { Minimize in } \nu: \quad \int_{\Lambda_{\alpha} \cup \Lambda_{\beta}} W(A) \mathrm{d} \nu(A)
$$

$$
\begin{gathered}
\nu=t \nu_{\alpha}+(1-t) \nu_{\beta} \text { commutes with determinant, } \\
\operatorname{supp}\left(\nu_{\gamma}\right) \subset \Lambda_{\gamma}, \quad \gamma=\alpha, \beta, \\
F=t \int_{\Lambda_{\alpha}} A \mathrm{~d} \nu_{\alpha}(A)+(1-t) \int_{\Lambda_{\beta}} A \mathrm{~d} \nu_{\beta}(A) .
\end{gathered}
$$

Notice how the integral constraint has been taken into account in the above decomposition for $\nu$.

Let us first examine the constraints. We introduce the following variables

$$
S_{\gamma}=\int_{\mathbf{R}^{2}}|\lambda|^{2} \mathrm{~d} \nu_{\gamma}^{(1)}(\lambda), \quad \gamma=\alpha, \beta
$$

where $\nu_{\gamma}^{(1)}$ is the probability measure resulting from the projection of $\nu_{\gamma}$ onto the first row variable. On the other hand if we let

$$
F_{\gamma}=\int_{\Lambda_{\gamma}} A \mathrm{~d} \nu_{\gamma}(A)
$$


we have that $F_{\gamma} \in \Lambda_{\gamma}$ (because $\Lambda_{\gamma}$ is a 2 -dimensional subspace) and $F=t F_{\alpha}+(1-t) F_{\beta}$. From these two conditions, it is immediate to obtain

$$
F_{\alpha}^{(1)}=\frac{1}{t(\beta-\alpha)}\left(\beta F^{(1)}+T F^{(2)}\right), \quad F_{\beta}^{(1)}=\frac{1}{(1-t)(\alpha-\beta)}\left(\alpha F^{(1)}+T F^{(2)}\right) .
$$

The important constraint on the commutation with det leads to the constraint

$$
\operatorname{det} F=t \int_{\Lambda_{\alpha}} \operatorname{det} A \mathrm{~d} \nu_{\alpha}(A)+(1-t) \int_{\Lambda_{\beta}} \operatorname{det} A \mathrm{~d} \nu_{\beta}(A)
$$

But notice that $\operatorname{det} A=\gamma\left|A^{(1)}\right|^{2}$ if $A \in \Lambda_{\gamma}$, so that by using (4.1),

$$
\operatorname{det} F=t \alpha S_{\alpha}+(1-t) \beta S_{\beta} .
$$

On the other hand, by Jensen's inequality,

$$
S_{\gamma} \geq\left|\int_{\mathbf{R}^{2}} \lambda \mathrm{d} \nu_{\gamma}^{(1)}(\lambda)\right|^{2}=\left|F_{\gamma}^{(1)}\right|^{2}
$$

and by using (4.2), we can write

$$
t^{2}(\beta-\alpha)^{2} S_{\alpha} \geq\left|\beta F^{(1)}+T F^{(2)}\right|^{2}, \quad(1-t)^{2}(\beta-\alpha)^{2} S_{\beta} \geq\left|\alpha F^{(1)}+T F^{(2)}\right|^{2} .
$$

After some algebra, we can rewrite the three restrictions as

$$
\begin{array}{r}
-\operatorname{det} F+t \alpha S_{\alpha}+(1-t) \beta S_{\beta}=0, \\
2 \beta \operatorname{det} F+\beta^{2}\left|F^{(1)}\right|^{2}+\left|F^{(2)}\right|^{2}-t^{2}(\beta-\alpha)^{2} S_{\alpha} \leq 0, \\
2 \alpha \operatorname{det} F+\alpha^{2}\left|F^{(1)}\right|^{2}+\left|F^{(2)}\right|^{2}-(1-t)^{2}(\beta-\alpha)^{2} S_{\beta} \leq 0 .
\end{array}
$$

Regarding the cost functional, we can rewrite it in terms of the $S$ variables as

$$
t S_{\alpha}+(1-t) S_{\beta}
$$

Altogether we face the mathematical programming problem

$$
\text { Minimize in } S=\left(S_{\alpha}, S_{\beta}\right): \quad t S_{\alpha}+(1-t) S_{\beta}
$$

subject to

$$
\begin{array}{r}
-\operatorname{det} F+t \alpha S_{\alpha}+(1-t) \beta S_{\beta}=0, \\
2 \beta \operatorname{det} F+\beta^{2}\left|F^{(1)}\right|^{2}+\left|F^{(2)}\right|^{2}-t^{2}(\beta-\alpha)^{2} S_{\alpha} \leq 0, \\
2 \alpha \operatorname{det} F+\alpha^{2}\left|F^{(1)}\right|^{2}+\left|F^{(2)}\right|^{2}-(1-t)^{2}(\beta-\alpha)^{2} S_{\beta} \leq 0 .
\end{array}
$$

where $\alpha, \beta$ are data of the initial problem, and $t$ and $F$ (and eventually $x \in \Omega$ ) are fixed, but arbitrary as the minimum value of this problem defines the relaxed integrand $C P W(F, t)$. A key issue here is whether the 


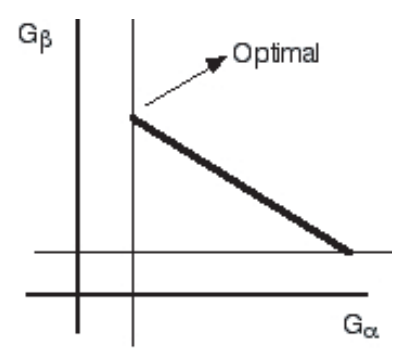

Figure 4.1. The feasible set.

optimal vector $S$ can be recovered from an admissible laminate $\nu$ in (4.1), and, in particular, we need to provide a precise way of going from $S$ to such a laminate.

Notice that this optimization problem is a linear, mathematical programming problem in the variables $S$. Three issues are important:

1. When is the admissible set non-empty?

2. Which are the points at which the minimum value is attained?

3. Which are the laminates for which the optimal $S$ are recovered?

The answer to the first two questions is elementary. Indeed, the admissible set will be non-empty provided that, for the point where the two inequality constraints become equalities, the constraint in the form of equality becomes non-positive. This is very easy to see geometrically (Fig. 4.1). The condition reads

$$
\alpha \beta(\alpha t+\beta(1-t))\left|F^{(1)}\right|^{2}+(\alpha(1-t)+\beta t)\left|F^{(2)}\right|^{2}-\left(t(1-t)(\beta-\alpha)^{2}+2 \alpha \beta\right) \operatorname{det} F \leq 0 .
$$

The second issue is also elementary. The admissible set (when it is non-empty) represents a segment with two extreme points (Fig. 4.1). The linear cost functional will attain its minimum on one of them, or become constant. After some elementary computations we obtain the expression

$$
C P W(F, t)=\frac{1}{t \beta(\beta-\alpha)}\left(\beta^{2}\left|F^{(1)}\right|^{2}+\left|F^{(2)}\right|^{2}-(\alpha t+\beta(2-t)) \operatorname{det} F\right)
$$

for the minimum value of the optimization problem for the variables $S$. The third question concerning whether these extreme points can be obtained as the second moments for appropriate measures $\nu_{\alpha}$ and $\nu_{\beta}$ as in (3.2) so that the convex combination $t \nu_{\alpha}+(1-t) \nu_{\beta}$ is a laminate, is a bit more involved. See [3,35] for details. We simply state the result for the vertex of the segment in Figure 4.1 when

$$
-\operatorname{det} F+\beta^{2}\left|F^{(1)}\right|^{2}+\left|F^{(2)}\right|^{2}-t^{2}(\beta-\alpha)^{2} S_{\alpha}=0
$$

Put

$$
\begin{array}{r}
g(A)=\alpha^{2} \beta^{2}\left|A^{(1)}\right|^{4}+\left|A^{(2)}\right|^{4}+\left(\alpha^{2}+6 \alpha \beta+\beta^{2}\right) \operatorname{det} A^{2} \\
-2 \alpha \beta\left|A^{(1)}\right|^{2}\left|A^{(2)}\right|^{2}-2 \alpha \beta(\alpha+\beta)\left|A^{(1)}\right|^{2} \operatorname{det} A-2(\alpha+\beta)\left|A^{(2)}\right|^{2} \operatorname{det} A,
\end{array}
$$


and

$$
\begin{aligned}
& r_{1}(A)=\frac{1}{2}+\frac{1}{2(\beta-\alpha) \operatorname{det} A}\left[\alpha \beta\left|A^{(1)}\right|^{2}-\left|A^{(2)}\right|^{2}-\sqrt{g(A)}\right], \\
& r_{2}(A)=\frac{1}{2}+\frac{1}{2(\beta-\alpha) \operatorname{det} A}\left[\alpha \beta\left|A^{(1)}\right|^{2}-\left|A^{(2)}\right|^{2}+\sqrt{g(A)}\right] .
\end{aligned}
$$

Take the matrices

$$
\begin{gathered}
A_{\alpha, t}=\left(\begin{array}{c}
z_{t} \\
\alpha T z_{t}
\end{array}\right), \quad z_{t}=\frac{1}{t} \frac{1}{\beta-\alpha}\left(\beta A^{(1)}+T A^{(2)}\right), \\
A_{\beta, i}=\left(\begin{array}{c}
w_{i} \\
\beta T w_{i}
\end{array}\right), \quad w_{i}=\frac{1}{1-r_{i}(A)} \frac{(-1)}{\beta-\alpha}\left(\alpha A^{(1)}+T A^{(2)}\right), \\
A_{\beta, j, t}=\left(\begin{array}{c}
w_{j, t} \\
\beta T w_{j, t}
\end{array}\right), \quad w_{j, t}=\frac{1}{t} \frac{r_{j}(A)}{1-r_{j}(A)} \frac{(-1)}{\beta-\alpha}\left(\alpha A^{(1)}+T A^{(2)}\right),
\end{gathered}
$$

where $i \neq j$ and $A^{(k)}$ stands for the $k$-th row of $A$. Finally, put

$$
s_{i, j}=\frac{\left(1-r_{i}(A)\right)\left[t\left(1-r_{j}(A)\right)-(1-t) r_{j}(A)\right]}{t\left(1-r_{j}(A)\right)-\left(1-r_{i}(A)\right) r_{j}(A)}, \quad i \neq j .
$$

Lemma 4.1 [35]. There are two second-order laminates supported in three matrices (except when $t=r_{i}(A)$ for $i=1$ or $i=2$ that the laminate collapses to afirst-order laminate) which are optimal microstructures. Namely, bearing in mindthe notation before the statement, the two laminates

$$
\nu_{i, j}=s_{i, j} \delta_{A_{\beta, i}}+\left(1-s_{i, j}\right)\left(\frac{t}{1-s_{i, j}} \delta_{A_{\alpha, t}}+\frac{1-s_{i, j}-t}{1-s_{i, j}} \delta_{A_{\beta, j, t}}\right),
$$

for $i \neq j$, where

$$
\begin{gathered}
\operatorname{det}\left(A_{\alpha, t}-A_{\beta, j, t}\right)=0 \\
\operatorname{det}\left(A_{\beta, i}-\frac{t}{1-s_{i, j}} A_{\alpha, t}-\frac{1-s_{i, j}-t}{1-s_{i, j}} A_{\beta, j, t}\right)=0,
\end{gathered}
$$

are optimal, and so are any convex combination of these two.

A similar result holds for the other extreme point of the segment in Figure 4.1.

When we put back the $x$-dependence, we have the following result.

Theorem 4.2.

$$
C P W(x, A, t)= \begin{cases}\varphi(A, t), & \text { if } \psi\left(A-G(x) \otimes e_{1}, t\right) \leq 0, \\ +\infty, & \text { else, }\end{cases}
$$

where $e_{1}=(1,0)$, and $\varphi(A, t)$ and $\psi(A, t)$ are explicitly given by

$$
\begin{aligned}
\varphi(A, t)= & \frac{1}{t \beta(\beta-\alpha)}\left(\beta^{2}\left|A^{(1)}\right|^{2}+\left|A^{(2)}\right|^{2}-(\alpha t+\beta(2-t)) \operatorname{det} A\right), \\
\psi(A, t)= & \alpha \beta(\beta(1-t)+\alpha t)\left|A^{(1)}\right|^{2}+(\alpha(1-t)+\beta t)\left|A^{(2)}\right|^{2} \\
& -\left(t(1-t)(\beta-\alpha)^{2}+2 \alpha \beta\right) \operatorname{det} A .
\end{aligned}
$$


In addition, optimal measures are given by

$$
\mu_{x, A, t}^{i, j}=\nu_{A-G(x) \otimes e_{1}, t}^{i, j}
$$

and

$$
\nu_{A, t}^{i, j}=s_{i, j} \delta_{A_{\beta, i}}+\left(1-s_{i, j}\right)\left(\frac{t}{1-s_{i, j}} \delta_{A_{\alpha, t}}+\frac{1-s_{i, j}-t}{1-s_{i, j}} \delta_{A_{\beta, j, t}}\right), \quad i \neq j,
$$

where we are using the formulae above, and e is the first vector of the canonical basis for $\mathbf{R}^{2}$.

If we put the auxiliary field $G$ back, where $\operatorname{div} G=g$, it is elementary to find the general form of the relaxed problem.

Corollary 4.3. The variational problem

$$
\text { Minimize in }(U, t): \quad I(U, t)=\int_{\Omega} \varphi(\nabla U(x), t(x)) \mathrm{d} x
$$

subject to

$$
\begin{aligned}
& U^{(1)}=u_{0} \quad \text { on } \quad \partial \Omega, \\
& \psi\left(\nabla U(x)-G(x) \otimes e_{1}, t(x)\right) \leq 0 \quad \text { a.e. } \quad x \in \Omega, \\
& \int_{\Omega} t(x) \mathrm{d} x \leq t_{0}
\end{aligned}
$$

admits optimal solutions.

The final step of our programme consists in checking whether minimizing sequences for the original optimal design problem can be built through the minimizers of this last problem and the underlying optimal measures. When integrands take on the value $+\infty$ abruptly, this is an important, delicate issue because generating sequences for gradient Young measures are not allowed to take on values off the finite set of the integrand, not even for very small sets. This has already been emphasized in Section 2. When an upper bound for integrands holds, this is not really an issue. For our particular problem this is indeed so (see Th. 2 in [3]).

\section{ANAlysis OF THE RELAXED PROBLEM AND SIMUlations}

The final step of our analysis is to explore the relaxed problem we have obtained at the end of the preceding section, namely

$$
\text { Minimize in }(U, t): \quad \int_{\Omega} \varphi(\nabla U(x), t(x)) \mathrm{d} x
$$

subject to

$$
\begin{gathered}
U \in H^{1}(\Omega), \quad U^{(1)}=u_{0} \text { on } \partial \Omega, \quad \psi(\nabla U(x), t(x)) \leq 0, \\
0 \leq t(x) \leq 1, \quad \int_{\Omega} t(x) \mathrm{d} x \leq t_{0}|\Omega|
\end{gathered}
$$

where again we have taken $G \equiv 0$ for simplicity.

This vector variational problem is convex (in the vectorial sense and taking into account the additional variable $t[25]$ ) and regular in the sense that it admits optimal solutions since it is a relaxation. Moreover, all functions involved are quadratic in the vector gradient variable and smooth. It is however a rather complex problem to analyze. 
One possibility is to look at optimality conditions introducing several multipliers to keep record of restrictions. This makes the problem more complicated precisely because of all the restrictions we have to enforce. Instead of this approach, we make the following simple but relevant observation. It is an elementary fact which tells a lot about the relationship between $\varphi, \psi$ and the quadratic cost $\left|F^{(1)}\right|^{2}$.

Lemma 5.1 [21]. For each $i=1,2$ and for fixed $t$, the optimal solution of the quadratic, mathematical programming problem

$$
\text { Minimize in } F^{(i)}: \quad \varphi(t, F)
$$

subject to $\psi(t, F) \leq 0$ occurs when

$$
(\alpha t+\beta(1-t)) F^{(1)}+T F^{(2)}=0 .
$$

In addition, the associated optimal structures (gradient Young measures) are first-order laminates with volume fraction $t$ for the $\alpha$-material and orientation of layers parallel to $F^{(1)}$. The optimal value in both cases is $\left|F^{(1)}\right|^{2}$.

The idea is then to replace the complicated constraint

$$
\psi(t(x), \nabla U(x)) \leq 0
$$

by the much simpler one

$$
(\alpha t(x)+\beta(1-t(x))) \nabla U^{(1)}(x)+T \nabla U^{(2)}(x)=0,
$$

hoping to keep track of the minimum we are seeking. But this last condition amounts, after all, to

$$
\operatorname{div}\left([\alpha t(x)+\beta(1-t(x))] \nabla U^{(1)}(x)\right)=0 \quad \text { in } \quad \Omega,
$$

and, as pointed out in the lemma, the cost simplifies to

$$
\int_{\Omega}\left|\nabla U^{(1)}(x)\right|^{2} \mathrm{~d} x
$$

If we remember that $U^{(1)}$ is our original field $u$, we are led to consider

$$
\text { Minimize in } t: \quad \int_{\Omega}|\nabla u(x)|^{2} \mathrm{~d} x
$$

subject to

$$
\begin{aligned}
\operatorname{div}([\alpha t(x)+\beta(1-t(x))] \nabla u(x)) & =0 \quad \text { in } \quad \Omega, \\
u & =u_{0} \quad \text { on } \partial \Omega, \\
0 \leq t(x) & \leq 1 \quad \text { in } \Omega, \\
\int_{\Omega} t(x) \mathrm{d} x & \leq t_{0}|\Omega| .
\end{aligned}
$$



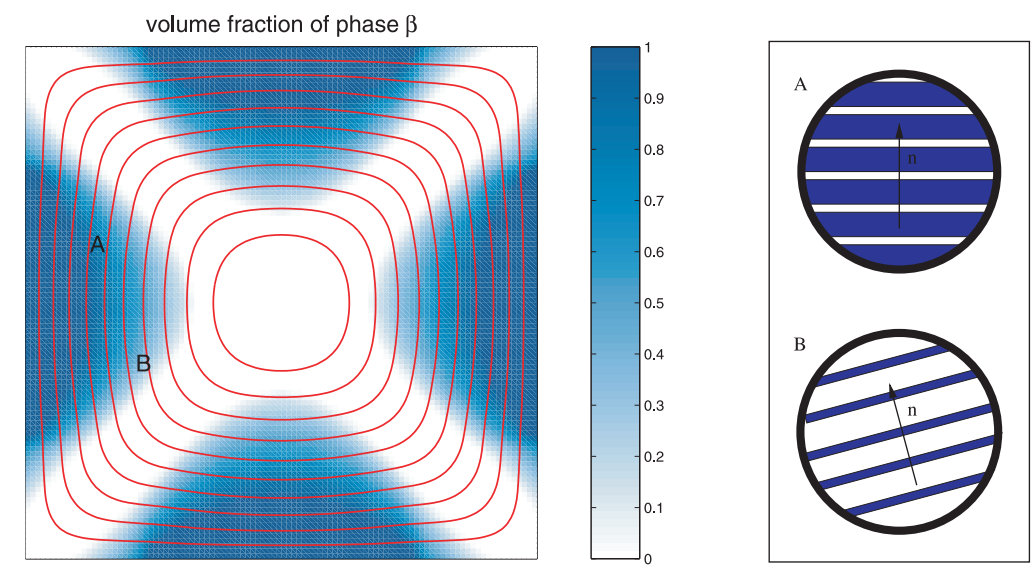

FiguRE 5.1.

Theorem 5.2 [37]. This last optimization problem admits optimal solutions $\tilde{t}$. In addition, optimal distributions are first-order laminates oriented parallel to $\nabla \tilde{u}$ if $\nabla \tilde{u}$ is the associated optimal field.

This fact is Tartar's result in [39] for a vanishing target field. We can now exploit optimality conditions for this new problem which are easily handled and in a standard way, to approximate optimal distributions of the two conducting materials. In all experiments we have performed the convergence was fine and the simulations stable and robust.

We show here several such simulations corresponding to a non-vanishing right-hand side $g$ in the equilibrium equation. All of these simulations have been performed by A. Donoso as part of his Ph.D. Thesis at Universidad de Castilla-La Mancha [22]

In all examples, the design domain is the unit square $\Omega=(0,1)^{2}$ and we take a vanishing boundary data $u_{0} \equiv 0$. The values for the parameters $\alpha$ and $\beta$ are 1 and 2 , respectively. Different simulations correspond to different right-hand sides $g$ or different values for the global resource constrain $t_{0}$. In addition, the grey (or color) level in these pictures indicates the optimal volume fraction $t(x)$ while the curves are level curves of the optimal, underlying electric field so that optimal microstructures are first order laminates with volume fraction $t(x)$ for the $\alpha$-material and layers align themselves orthogonal to those level curves (parallel to the field). We have tried to indicate this in the two points $A$ and $B$ of Figure 5.1. Finally, the strong convergence of minimizing fields to optimal fields is due to the fact that the optimal underlying gradient Young measures (first-order laminates) reduce to a Dirac delta measure in the first row (see [34].

The first set of simulations are those in [30]. In the first example (Fig. 5.1), the right-hand side $g$ is taken to be identically 1 and $t_{0}=0.4$.

The next two cases correspond to

$$
g(x)= \begin{cases}1, & \text { if } x \in(1 / 4,3 / 4)^{2} \\ 0, & \text { else. }\end{cases}
$$

Optimal structures are given in Figure 5.2 for the case $t_{0}=0.15$ and in Figure 5.3 for $t_{0}=0.4$. 


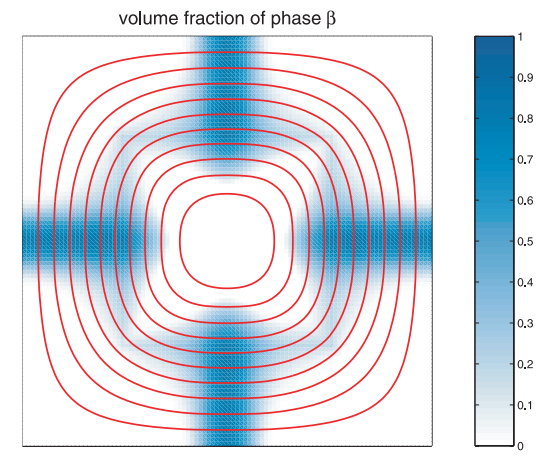

FiguRe 5.2.

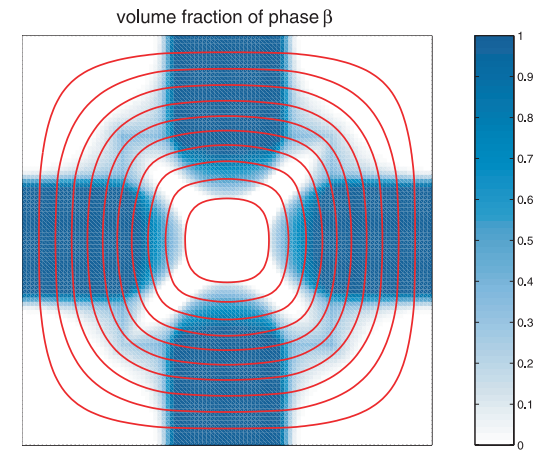

FiguRE 5.3.

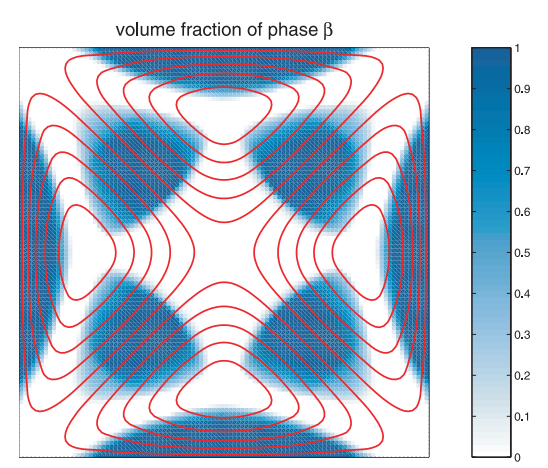

FiguRE 5.4.

Finally, for Figures 5.4 and 5.5 we have

$$
g(x)=\left(x_{1}-\frac{1}{2}\right)^{2}-\left(x_{2}-\frac{1}{2}\right)^{2}, \quad g(x)=\mathrm{e}^{-100\left(x_{1}-0.5\right)^{2}}+\mathrm{e}^{-100\left(x_{2}-0.5\right)^{2}}
$$

respectively. In both cases $t_{0}=0.4$.

More simulations can be found in [22]. 


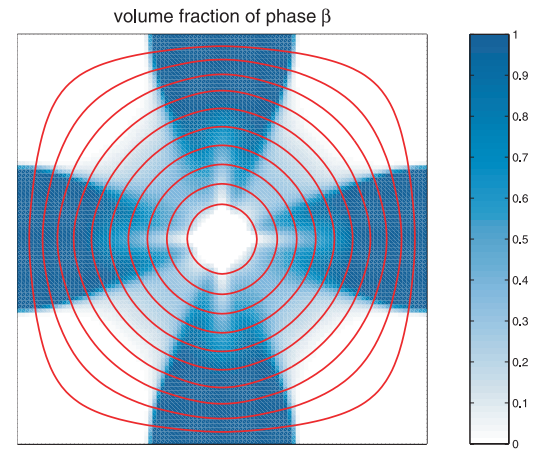

FiguRe 5.5.

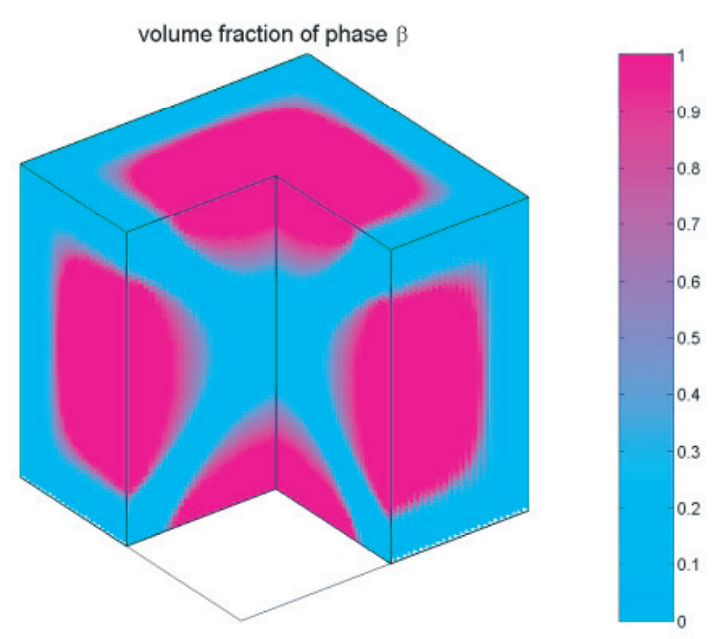

FiguRE 6.1.

\section{The THREE-DIMENSIONAL CASE}

The same analysis as the one described here can be carried out for the three dimensional situation although the analysis is somewhat more complex and, most definitely, simulations are. The computation of the relaxed integrands were computed in [11] while the computations will appear in [23]. As an illustration we have taken two examples from this last reference.

The first example (Fig. 6.1) corresponds to taking the same values for $\alpha, \beta$, the right-hand side in the equilibrium equation $g$, and the global-resource-constraint parameter $t_{0}$, as in the two-dimensional examples, namely, $\alpha=1,=\mathbf{2}, g \equiv 1$, and $t_{0}=0.4$. The domain is the unit cube in $\mathbf{R}^{3}$ and we always take a vanishing boundary condition for the field equation.

For the second example (Fig. 6.2), only the function $g$ changes. This time

$$
g(x)=\mathrm{e}^{-100|x-0.5|^{2}}
$$

Acknowledgements. This work is supported by BFM2001-0738 of MCyT (Spain)and by GC-02-001 of JCCM (Castilla-La Mancha). 


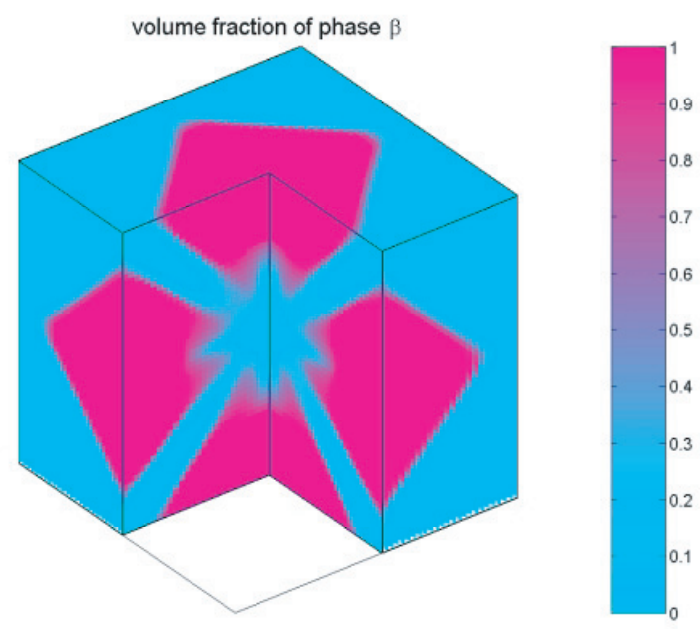

FiguRE 6.2 .

\section{REFERENCES}

[1] G. Allaire, Shape optimization by the homogenization method. Springer (2002).

[2] S. Antman, Nonlinear Problems of Elasticity. Springer (1995).

[3] E. Aranda and P. Pedregal, Constrained envelope for a general class of design problems. DCDS-A, Supplement Volume 2003 (2002) 30-41.

[4] E.J. Balder, Lectures on Young Measures. Cahiers de Mathématiques de la Décision No. 9517, CEREMADE, Université Paris IX (1995).

[5] J.M. Ball, Convexity conditions and existence theorems in nonlinear elasticity. Arch. Rat. Mech. Anal. 63 (1977) 337-403.

[6] J.M. Ball, A version of the fundamental theorem for Young measures, PDE's and continuum models of phase transitions, M. Rascle, D. Serre and M. Slemrod Eds. Springer. Lect. Notes Phys. 344 (1989) 207-215.

[7] J.M. Ball, Some open problems in elasticity, in Geometry, Mechanics and Dynamics, P. Newton, P. Holmes, A. Weinstein Eds. Springer (2002) 3-59.

[8] J.M. Ball and R.D. James, Finephase mixtures as minimizers of energy. Arch. Rat. Mech. Anal. 100 (1987) 13-52.

[9] J.M. Ball and F. Murat, Remarks on Chacon'sbiting lemma. Proc. AMS 107 (1989) 655-663.

[10] K. Battacharya and G. Dolzmann, Relaxation of some multi-well problems. Proc. Roy. Soc. Edinb. 131A (2001) $279-320$.

[11] J.C. Bellido, Explicit computation of the relaxed density coming from a three-dimensional optimal design problem. Non-Lin. Anal. TMA 52 (2002) 1709-1726.

[12] J.C. Bellido and P. Pedregal, Explicit quasiconvexification of some cost functionals depending on derivatives of the state in optimal design. Disc. Cont. Dyn. Syst. A 8 (2002) 967-982.

[13] M.P. Bendsoe, Optimization of structural topology, shape and material. Springer (1995).

[14] M. Bousselsal and M. Chipot, Relaxation of some functionals of the calculus of variations. Arch. Math. 65 (1995) 316-326.

[15] M. Bousselsal and R. Le Dret, Remarks on the quasiconvex envelope of some functions depending on quadratic forms. Boll. Union. Mat. Ital. Sez. B 5 (2002) 469-486.

[16] M. Bousselsal and R. Le Dret, Relaxation of functionals involving homogeneous functions and invariance of envelopes. Chinese Ann. Math. Ser. B 23 (2002) 37-52.

[17] L. Carbone and R. De Arcangelis, Unbounded functionals in the Calculus of Variations, Representation, Relaxation and Homogenization, Chapman and Hall. CRC, Monographs and Surveys in Pure and Applied Mathematics. Boca Raton, Florida $125(2002)$

[18] P.G. Ciarlet, Mathematical Elasticity, Vol. I: Three-dimensional Elasticity. North-Holland, Amsterdam (1987).

[19] B. Dacorogna, Direct methods in the Calculus of Variations. Springer (1989).

[20] G. Dolzmann, B. Kirchheim, S. Muller and V. Sverak, The two-well problem in three dimensions. Calc. Var. 10 (2000) 21-40.

[21] A. Donoso and P. Pedregal, Optimal design of 2-d conducting graded materials by minimizing quadratic functionals in the field. Struct. Opt. (in press) (2004).

[22] A. Donoso, Optimal design modelled by Poisson's equation in the presence of gradients in the objective. Ph.D. Thesis, Univ. Castilla-La Mancha (2004).

[23] A. Donoso, Numerical simulations in 3-d heat conduction: minimizing the quadratic mean temperature gradient (2004), submitted. 
[24] D. Faraco, Beltrami operators and microstructure. Ph.D. Thesis, University of Helsinki (2002).

[25] I. Fonseca, D. Kinderlehrer and P. Pedregal, Energy functionals depending on elastic strain and chemical composition. Calc. Var. 2 (1994) 283-313.

[26] Y. Grabovsky, Optimal design problems for two-phase conducting composites with weakly discontinuous objective functionals. Adv. Appl. Math 27 (2001) 683-704.

[27] D. Kinderlehrer and P. Pedregal, Gradient Young measures generated by sequences in Sobolev spaces. J. Geom. Anal. 4 (1994) 59-90.

[28] R. Kohn, The relaxation of a double-well energy. Cont. Mech. Thermodyn. 3 (1991) 193-236.

[29] R.V. Kohn and G. Strang, Optimal design and relaxation of variational problems, I, II and III. CPAM 39 (1986) 113-137, 139-182 and 353-377.

[30] R. Lipton and A. Velo, Optimal design of gradient fields with applications to electrostatics, in Nonlinear Partial Differential Equations Appl., College de France Seminar, D. Cioranescu, F. Murat and J.L Lions Eds. Chapman and Hall/CRCResearch Notes in Mathematics (2000).

[31] Ch.B. Morrey, Quasiconvexity and the lower semicontinuity of multiple integrals. Pacific J. Math. 2 (1952) $25-53$.

[32] Ch.B. Morrey, Multiple Integrals in the Calculus of Variations. Berlin, Springer (1966).

[33] P. Pedregal, Parametrized Measures and Variational Principles. Birkhäuser, Basel (1997).

[34] P. Pedregal, Variational methods in nonlinear elasticity. SIAM, Philadelphia (2000).

[35] P. Pedregal, Constrained quasiconvexification of the square of the gradient of the state in optimal design. QAM 62 (2004) 459-470.

[36] P. Pedregal, Optimal design in 2-d conductivity for quadratic functionals in the field, in Proc. NATO Advan. Meeting Non-lin. Homog., Warsaw, Poland, Kluwer (2004) 229-246.

[37] P. Pedregal, Optimal design in two-dimensional conductivity for a general cost depending on the field. Arch. Rat. Mech. Anal. (2004) (in press).

[38] Y. Reshetnyak, General theorems on semicontinuity and on convergence with a functional. Sibir. Math. 8 (1967) $801-816$.

[39] L. Tartar, Remarks on optimal design problems, in Calculus of Variations, Homogenization and Continuum Mechanics, G. Buttazzo, G. Bouchitte and P. Suquet Eds. World Scientific, Singapore (1994) 279-296.

[40] L. Tartar, An introduction to the homogenization method in optimal design, Springer. Lect. Notes Math. 1740 (2000) 47-156. 